Holmes RD. Insufficient evidence for the role of school dental screening in improving oral health. Evidence-Based Dentistry 2018, 19(1), 3-4.

DOI link

https://doi.org/10.1038/sj.ebd.6401281

ePrints link

http://eprint.ncl.ac.uk/245715

Date deposited

$27 / 03 / 2018$

Embargo release date

$23 / 09 / 2018$

Copyright

This is the authors' accepted manuscript of an article that has been published in its final definitive form by Nature Publishing Group, 2018 


\author{
Summary \\ (evidence level 1A ) Review / Oral Health \\ Insufficient evidence for the role of school dental screening in improving oral health
}

\title{
Abstracted from
}

Arora A, Khattri S, Ismail NM, Kumbargere Nagraj S, Prashanti E. School dental screening programmes for oral health. Cochrane Database of Systematic Reviews 2017, Issue 12. Art. No.: CD012595. DOI: 10.1002/14651858.CD012595.pub2.

Address for correspondence Luisa Fernandez Mauleffinch, Managing Editor, Cochrane Oral Health Group, School of Dentistry, The University of Manchester, JR Moore Building, Oxford Road, Manchester, M13 9PL, UK. E-mail:luisa.fernandez@manchester.ac.uk

This paper is based on a Cochrane Review published in the Cochrane Library 2017, issue 12 (see www.thecochranelibrary.com for information). Cochrane Reviews are regularly updated as new evidence emerges and in response to feedback, and the Cochrane Library should be consulted for the most recent version of the review.

Question Does school dental screening improve oral health?

Data sources The Cochrane Oral Health's Trials Register, the Cochrane Central Register of Controlled Trials (CENTRAL), Medline, Embase, the US National Institutes of Health Trials Registry (ClinicalTrials.gov) and the World Health Organization International Clinical Trials Registry Platform databases.

Study selection Randomised controlled trials (cluster or parallel) Evaluating school dental screening compared with no intervention or with one type of screening compared with another were included.

Data extraction and synthesis Two reviewers independently abstracted data and assessed risk of bias. Risk ratios were calculated for dichotomous outcomes with data being polled where appropriate. The GRADE approach was used to interpret findings.

Results 6 trials involving 19498 children were included. Two were considered to be at low risk of bias, three at unclear risk and one at high risk. No conclusions could be made from 4 studies comparing traditional screening versus no screening because the evidence was inconsistent. Two trials evaluating criteria-based screening versus no screening suggested a possible benefit $\mathrm{RR}=1.07$ ( $95 \% \mathrm{Cl} ; 0.99$ to 1.16$)$. No difference was found when comparing criteria-based screening with traditional screening, $R R=1.01,(95 \% \mathrm{Cl} ; 0.94$ to 1.08$)$. No trials reported on longterm follow up or cost-effectiveness and adverse events.

Conclusions The trials included in this review evaluated short-term effects of screening, assessing follow-up periods of three to eight months. We found very low certainty evidence that was insufficient to allow us to draw conclusions about whether there is a role for traditional school dental screening in improving dental attendance. For criteria-based screening, we found 
low-certainty evidence that it may improve dental attendance when compared to no screening. However, when compared to traditional screening there was no evidence of a difference in dental attendance (very low-certainty evidence).

We found low-certainty evidence to conclude that personalised or specific referral letters improve dental attendance when compared to non-specific counterparts. We also found lowcertainty evidence that screening supplemented with motivation (oral health education and offer of free treatment) improves dental attendance in comparison to screening alone.

We did not find any trials addressing cost-effectiveness and adverse effects of school dental screening.

\section{Commentary}

This recently published systematic review by Arora et al. adhered to the standard methodological processes used by Cochrane and it included electronic searches of some databases as recently as mid-March 2017. The authors placed no restrictions upon language or publication status. Children and adolescents aged 3-19 years attending school from any country were eligible for inclusion. Randomised controlled trials (RCTs) were included whether the unit of randomisation was either individual children or a group (e.g. a school or class). Data extraction followed the Cochrane Handbook for Systematic Reviews of Interventions. ${ }^{1}$ An assessment of risk of bias in the included studies resulted in a low, unclear or high risk of bias judgement across a number of domains. ${ }^{2}$

From the 2238 records initially identified, 25 full-text articles were assessed for eligibility. Six RCT studies ultimately satisfied the inclusion criteria and were incorporated in the quantitative synthesis (meta-analysis). The six studies varied considerably in relation to how test-positive children were identified, followed-up and referred. The follow-up period of the included trials was less than 2 years so the authors were only able to report the short-term effects of school dental screening. A further limitation is that the main outcome measure was reported attendance or registration with a dentist. Only one study included the prevalence of dental caries per child and associated clinical variables. ${ }^{3}$

Differences in the screening interventions meant that only four studies could be included in a meta-analysis assessing 'traditional screening versus no screening' with dental attendance as the outcome. In this case, an inconclusive result was found with very low certainty evidence. The high heterogeneity may be partly explained by the fact that one study was an individuallevel RCT and the other three were cluster-RCTs.

Criteria-based screening (the referring dentist using pre-established criteria) showed a 7\% relative increase in dental attendance compared to no screening but the confidence interval ranged from a $1 \%$ decrease to a $16 \%$ increase and there was low certainty of the evidence underlying the effect estimate. There was no evidence of a difference between criteria-based screening compared to traditional screening.

Within this systematic review, one of the included papers studied the effectiveness of different types of referral letter used following screening, but it is worth highlighting that this paper focused upon their effectiveness following orthodontic screening. ${ }^{4}$ A specific (personalised) 
screening letter was preferred by participants to a non-specific version. The risk ratio (RR) was 1.39 (95\% $\mathrm{Cl} ; 1.09$ to 1.77$)$ indicating a $39 \%$ relative increase in attendance to a general dentist in the specific referral group versus the non-specific group but there was low certainty of the underlying evidence. Elsewhere within this review, Hebbal (2005) found that traditional school screening with additional motivation (e.g. oral health education) compared to traditional school screening alone, led to a $208 \%$ relative increase in dental attendance RR $3.08(95 \% \mathrm{Cl} ; 2.57$ to 3.71) again, with low certainty evidence. ${ }^{5}$ However, the improved attendance in the group with additional motivation cannot necessarily be associated to the effect of screening per se.

There are a number of limitations with the studies included in this systematic review and identified by the authors. 1) The included trials were all short-term and it is not known if the benefits of screening continue beyond this. 2) 'Dental attendance' as an outcome measure does not provide evidence that better oral health was achieved in those referred. 3) There is a need for standardisation of terminology (for example, when authors discuss using 'specific' referral letters and 'criteria-based' screening) to make interventions more transparent for study comparisons and for readers. 4) None of the RCTs reported data on any adverse effects of school dental screening. 5) None of the RCTs assessed the cost-effectiveness of the interventions used. 6) The conduct and reporting of clinical trials should adhere to CONSORT group guidelines. ${ }^{6}$

It is reassuring though not surprising, that the key results of the meta-analysis by Arora et al. published in late 2017 are in agreement with the earlier systematic review by Joury et al. published online in late 2016. ${ }^{7}$ (My commentary on the Joury et al. paper appeared in EBD in October 2017). ${ }^{8}$ There is no evidence of improvement in dental attendance or reduction in dental caries between 'screening' and 'no screening' groups, despite a slight difference with the studies included in each systematic review. Both systematic reviews found low to very low certainty in the underlying evidence. Beyond this, it is not clear if improved dental attendance in those screened positive translates to improved oral health.

Richard D Holmes Centre for Oral Health Research, Newcastle University, Newcastle upon Tyne, UK

$1 \quad$ Higgins JPT, Deeks JJ (editors). Chapter 7: Selecting studies and collecting data. In: Higgins JPT, Green S (editors), Cochrane Handbook for Systematic Reviews of Interventions Version 5.1.0 (updated March 2011). The Cochrane Collaboration, 2011. Available from handbook.cochrane.org (Accessed January 2018).

2 Higgins JPT, Altman DG, Sterne JAC (editors). Chapter 8: Assessing risk of bias in included studies. In: Higgins JPT, Green S (editors), Cochrane Handbook for Systematic Reviews of Interventions Version 5.1.0 (updated March 2011). The Cochrane Collaboration, 2011. Available from handbook.cochrane.org (Accessed January 2018).

3 Milsom K, Blinkhorn A, Worthington $\mathrm{H}$, Threlfall A, Buchanan K, Kearney-Mitchell P, Tickle $M$. The effectiveness of school dental screening: a cluster-randomized control trial. J Dent Res 2006; 85: 924-928.

4 Burden DJ, Mitropoulos CM. Effectiveness of a personalised referral letter following orthodontic screening. Comm Dent Oral Epidemiol 1994; 22: 323-326. 
$5 \quad$ Hebbal M, Nagarajappa R. Does school-based dental screening for children increase follow-up treatment at dental school clinics? J Dent Educ 2005; 69: 382-386.

6 Schulz KF, Altman DG, Moher D, for the CONSORT Group. CONSORT 2010 Statement: updated guidelines for reporting parallel group randomised trials. BMJ 2010; 340: c332

7 Joury E, Bernabe E, Sabbah W, Nakhleh K, Gurusamy K. Systematic review and metaanalysis of randomised controlled trials on the effectiveness of school-based dental screening versus no screening on improving oral health in children. J Dent 2017; 58: 110.

8 Holmes RD. No evidence to support or refute the benefits of school dental screening. [Commentary]. Evid Based Dent 2017; 18: 66-67. 